\title{
Results of urinary dissolution therapy for radiolucent calculi
}

\author{
Maneesh Sinha, Kumar Prabhu, Prasanna Venkatesh, Venkatesh Krishnamoorthy \\ Department of Urology NU Hospitals, Bangalore, India
}

\section{ABSTRACT}

Purpose: In this paper we present our experience with dissolution therapy of radiolucent calculi.

Materials and Methods: This was a retrospective analysis of patients who were offered urinary dissolution therapy between January 2010 and June 2011. Patients were treated with tablets containing potassium citrate and magnesium oxide. Partial dissolution was defined as at least a 50\% reduction in stone size. Patients with complete or partial dissolution were classified in the successful dissolution group. Patients with no change, inadequate reduction, increase in stone size and those unable to tolerate alkali therapy were classified as failures. Patient sex, stenting before alkalinization, stone size, urine $\mathrm{pH}$ at presentation and serum uric acid levels were analyzed using Fisher t-test for an association with successful dissolution.

Results: Out of 67, 48 patients reported for follow up. 10 (15\%) had complete dissolution and 13 (19\%) had partial dissolution. Alkalinization was unsuccessful in achieving dissolution in 25 (37\%). Stenting before alkalinization, patient weight ( $<60$ vs. $>75 \mathrm{~kg}$ ) and serum uric acid levels ( $\leq 6$ vs. $>6$ ) were the only factors to significantly affected dissolution rates $(\mathrm{p}=0.039, \mathrm{p} 0.035, \mathrm{p} 0.01$ respectively).

Conclusions: A policy of offering dissolution therapy to patients with radiolucent calculi had a successful outcome in $34 \%$ of patients.

\section{ARTICLE INFO}

\section{Key words:}

Urolithiasis; Uric Acid;

Therapy; Calculi; Urinary Tract

Int Braz J Urol. 2013; 39: 103-7

Submitted for publication:

May 17, 2012

Accepted after revision: October 16, 2012

\section{INTRODUCTION}

Pure uric acid calculi offer the urologist a unique opportunity to manage stones medically. Factors implicated in uric acid stone formation include a persistently acidic urine, dehydration causing low urine volumes and hyperuricosuria. Of these, alteration in urinary $\mathrm{pH}$ appears to be the most important contributory mechanism. Urinary dissolution therapy for management of uric acid calculi would currently qualify as standard of care (1). In an outpatient setting, however, it is rare to find a patient with a proven uric acid calculus. The only sure way of diagnosing an uric acid calculus is by doing a stone analysis, which, due to obvious reasons, is not feasible. While CT Honsefield density and stone density to stone size can suggest the presence of an uric acid calculus, non-contrast CT is not a routine investigation at our institution. A practical surrogate is to treat radiolucent calculi with urinary alkalinization.

Potassium citrate and sodium bicarbonate are commonly used for alkalinization. Potassium citrate is preferable as potassium urate is more soluble than sodium urate and the addition of sodium results in a concomitant increase in sodium (2). Alkalinization using intravenous lactate and contact chemolysis using irrigation of the pelvicalyceal system with tromethamine or sodium bicarbonate are effective options (3). The success of oral chemolysis has rendered these approaches obsolete. At our hospital we have been advocating oral dissolution therapy for patients presenting with radiolucent stones. We present an audit of this practice. 


\section{MATERIALS AND METHODS}

This was a retrospective analysis done after obtaining approval from our ethics committee. Patients who were offered urinary dissolution therapy between January 2010 and June 2011 at our institution were included. Patients with calculi noted on ultrasound but not seen on an X-ray KUB were included in the study. Affected systems were stented before alkalinization in the presence of stones causing obstruction, febrile urinary tract infection, renal failure or recurrent pain. Patients were started on $978 \mathrm{mg}$ tablets of potassium magnesium citrate. Each tablet contained $7 \mathrm{mEq}$ of potassium, $3.5 \mathrm{mEq}$ of magnesium and $10.5 \mathrm{mEq}$ of citrate.

Emphasis was laid on taking the tablets with an empty stomach ensuring a gap of at least two hours between meal times and tablets. The initial dose was two tablets three times a day. All patients were taught home monitoring of morning urine using a $\mathrm{pH}$ strip. Patients reported urinary $\mathrm{pH}$ on phone after 7 days. Drug dosage was adjusted to maintain urine $\mathrm{pH}$ between 6 and 7. Compliance with treatment and urinary $\mathrm{pH}$ monitoring results were self reported.

Ultrasonography was advised after one to six months on alkali therapy to assess stone size. Partial dissolution was defined as at least a 50\% reduction in stone size. Patients with complete or partial dissolution were classified in the successful dissolution group. Patients with no change, inadequate reduction, increase in stone size and those refusing to take the tablets due to upper gastrointestinal side effects were classified as failures. Patient sex, stenting before alkalinization, stone size, urine $\mathrm{pH}$ at presentation and serum uric acid levels were analyzed using Fisher t-test to look for an association with successful dissolution.

\section{RESULTS}

All patients underwent an initial evaluation with routine blood chemistry, ultrasonography and an x-ray KUB. 67 patients with radiolucent calculi were offered urinary alkalinization. Of these, 48 (71\%) had an adequate follow-up. Ten (15\%) had complete dissolution and 13 (19\%) had partial dissolution. Alkalinization was unsuccessful in achieving dissolution in 25 (37\%) (Table-1). Patients were taught domiciliary monitoring of urinary $\mathrm{pH}$ using $\mathrm{pH}$ strips. Urinary alkalinization was achieved as early as three days after starting therapy. Two tablets 3 times a day achieved adequate alkalinization in 39 patients. Five patients required dose reduction to one tablet 3 times a day. Three patients required dose escalation to 9 tablets a day and one patient who eventually had successful dissolution needed 15 tablets a day. The mean duration at which stone clearance was reported was $103.6 \pm 89$ days. In the group that underwent successful dissolution stone bulk was larger (17.45 vs. $14.8 \mathrm{~mm}$ ), patients weighed less (58 vs. $68.9 \mathrm{~kg}$ ), uric acid levels were lower (4.9 vs. 6.22) and urine was more acidic at presentation (5.59 vs. 5.90) (Table-2). However, on applying the Fisher single tailed exact t-test, stenting before alkalinization, patient weight $(<60$ vs. $>75 \mathrm{~kg}$ ) and serum uric acid levels $(\leq 6$ vs. $>6)$ were the only factors to significantly affected dissolution rates $(\mathrm{p}=0.039$, p 0.035, p 0.01 respectively). Stone size ( $\leq 10 \mathrm{~mm}$

Table 1 - Dissolution rates and stone sizes before and after treatment in each group.

\begin{tabular}{lccc}
\hline Group & $\mathrm{n}(\%)$ & Pre Rx size $(\mathrm{mm})$ & Post Rx size $(\mathrm{mm})$ \\
\hline Complete Dissolution & $10(15 \%)$ & 15.67 & 0 \\
Partial Dissolution & $13(19 \%)$ & 18.9 & 6.74 \\
No change/ increase & $25(37 \%)$ & 14.8 & 15.9 \\
Lost to follow up & $19(29 \%)$ & 10.9 & - \\
\hline
\end{tabular}


Table 2 - Statistical significance of factors affecting dissolution.

\begin{tabular}{lc}
\hline Factors & P value \\
\hline Stented vs nonstented & 0.039 \\
Weight $<60$ vs. $>75$ & 0.035 \\
Serum uric acid $(\leq \mathrm{vs} .>6 \mathrm{mg} / \mathrm{dL})$ & 0.01 \\
Stone size $\leq 10$ vs. $>10 \mathrm{~mm}$ & 0.136 \\
Urine $\mathrm{pH} \leq 5.5$ vs. $\geq 6$ & 0.11 \\
Sex & 0.07 \\
\hline
\end{tabular}

vs. $>10 \mathrm{~mm})$, urine $\mathrm{pH}$ at presentation $(\leq 5.5 \mathrm{vs} . \geq$ 6) of and sex of the patient did not affect success rates (p 0.14, 0.11 and 0.07 respectively) (Table-3). Stone analysis was available in 6 patients who underwent surgical intervention following failure of dissolution. Five of these had predominantly calcium oxalate stones. One patient had a stone composed of $80 \%$ uric acid and $20 \%$ calcium oxalate dihydrate.

\section{DISCUSSION}

Urinary dissolution for uric acid stones is now standard therapy. In a clinical scenario, however, the stone analysis is usually unavailable. Pointers which suggest the presence of uric acid stones include radiolucency on an X-ray KUB, a CT Hounsefield density of $200-400$ or a ratio of stone density to stone size of $<80 \mathrm{HU} / \mathrm{mm}(4,5)$. At our institution ultrasonography and x-ray KUB continue to remain the mainstays of diagnosis. Stones which are evident on ultrasonography but not seen on a plain radiograph are offered dissolution therapy based on a surrogate diagnosis of possible uric acid urolithiasis. In this paper we present an audit of our practice.

Consumption of a typically vegetarian diet prevalent in this region results in alkalinization of urine. A careful history of meal timings and daily routines was taken. Patients were advised to take their tablets at least two hours before or after meals. Urine $\mathrm{pH}$ however was monitored only once a day. As far as feasible, patients were advised to check the first voided sample. A magnesium containing formulation was used due to the known additional inhibitory effects of magnesium on stone formation. We acknowledge that it is not a necessary ingredient if the only goal is urinary alkalinization.

The first major hurdle faced in dissolution therapy was compliance. Almost one-third of our patients were lost to follow-up after being started on therapy. Of those who did remain on follow-up many had to be repeatedly persuaded to continue with drug therapy. The size of the tablets was a constant complaint by patients who baulked at the idea of taking two large tablets 3 times a day. Pharmaceutical research into once a day dosing will be a significant step in improving compliance. As most patients who are started on dissolution therapy are those who are asymptomatic and without any complications, an extra degree of motivation is

Table 3 - Comparison of baseline factors in patients undergoing successful dissolution and failures.

\begin{tabular}{lcc}
\hline Factor & $\begin{array}{c}\text { Mean value in patients with } \\
\text { successful dissolution }\end{array}$ & Mean values in failures \\
\hline Stone size $(\mathrm{mm})$ & 17.45 & 14.8 \\
Patient weight $(\mathrm{kg})$ & 58 & 68.9 \\
Urine pH at presentation & 5.59 & 6.90 \\
Serum uric acid $(\mathrm{mg} / \mathrm{dL})$ & 4.9 & 6.22 \\
\hline
\end{tabular}


essential in continuing treatment. We believe that the only plausible explanation of the better results seen in patients who had undergone stenting is that these symptomatic patients were more compliant with their treatment.

An important question is the duration for which therapy needs to be given. Trincheri et al. have reported complete dissolution in 5 out of 8 patients after treatment ranging from 6 weeks to 6 months (6). A wide variation is evident in our results where stone clearance was reported at 103.6 \pm 89 days. We recommend continuation of alkalinization for at least 6 months in patients who show a partial dissolution.

Although statistically not significant, larger stones seemed to respond better to therapy. This, we believe, was more attributable to the lower sensitivity of plain radiographs at smaller stone sizes than the actual ability of treatment to achieve dissolution. The sensitivity of plain radiographs of the KUB region has been reported to be between $48-63 \%(7,8)$. Smaller calcium stones are more likely to be missed on $\mathrm{x}$-rays and will obviously not respond to urinary alkalinization. Where stone analysis was available, failures were noted in patients with calcium bearing stones. Thus a potential drawback of our approach of offering dissolution to patients with radiolucent calculi is the invariable inclusion of a proportion of patients with calcium stones who are unlikely to respond.

Better results with lower uric acid levels can be explained on the basis of possible renal hypouricaemia in our patient population. Mutations in URAT1 and SCL2A9 result in decreased reabsorption in the proximal tubule. This results in hyperuricosuria along with low or normal serum uric acid levels (7-9). Similar mechanisms may contribute to idiopathic uric acid nephrolithiasis. This would explain a higher likelihood of uric acid stones in patients with lower serum levels and therefore a better response to dissolution therapy.

As compared to lean stone formers a higher prevalence of uric acid stones has been reported in the obese $(10,11)$. Maalouf et al. have reported a linear decrease in urine $\mathrm{pH}$ with an increasing weight in stone formers (12). Taylor has also reported an inverse relationship between BMI and urine $\mathrm{pH}$ (13). These facts seem to be in direct conflict with the better dissolution rates seen in patients with lower weight in our study. Other metabolic abnormalities including a higher incidence of hypocitraturia, larger potassium citrate requirements and non-compliance with other dietary restrictions may explain the poorer outcomes in the obese.

Technical factors such as skin to stone distance as well as operator experience affect the results of ultrasonography as a diagnostic and monitoring tool in urolithiasis. Recent studies reporting sensitivity varying from $40-73 \%$ reflect these limitations of ultrasonography (14-16). Unenhanced CT scans, even with low dose protocols, result in a significant radiation exposure rendering them impractical for monitoring stone size (17). Our protocol has been dependent on a single, experienced radiologist, who has followed up all our patients. The results of this study need to be viewed in this context.

The intent of the paper was to audit our established clinical protocol. While the quantitative excretion of uric acid and the volume of urine affect uric acid stone formation, the most important factor is urinary $\mathrm{pH}$ (18). All patients were encouraged to increase their water intake to at least 2 litres/day. Measurement of urinary output would have been a desirable study parameter. However, compliance issues make its applicability questionable in the routine clinical scenario. Urinary uric acid measurements could have altered the treatment algorithm by helping to decide on the need for xanthine oxidase inhibitors. Our protocol limits itself to monitoring the most important parameter, namely urinary $\mathrm{pH}$. We do not routinely do urine cultures before starting alkalinization.

\section{CONCLUSIONS}

A policy of offering dissolution therapy to patients with radiolucent calculi had a successful outcome in 34\% of patients. Compliance was a major issue with $29 \%$ of patients failing to follow-up after an initial visit. This approach invariably includes patients with calcium bearing stones. However, the attractiveness and safety of dissolution therapy justifies its use in patients with radiolucent calculi. 
We would recommend a trial of urinary alkalinization in asymptomatic patients where ultrasonography diagnoses urolithiasis which is not seen on a good quality radiograph.

\section{CONFLICT OF INTEREST}

None declared.

\section{REFERENCES}

1. Becker G; Caring for Australians with Renal Impairment (CARI): The CARI guidelines. Kidney stones: uric acid stones. Nephrology (Carlton). 2007; 12(Suppl 1): S21-5.

2. Federle MP, McAninch JW, Kaiser JA, Goodman PC, Roberts J, Mall JC: Computed tomography of urinary calculi. AJR Am J Roentgenol. 1981; 136: 255-8.

3. Nakada SY, Hoff DG, Attai S, Heisey D, Blankenbaker D, Pozniak M: Determination of stone composition by noncontrast spiral computed tomography in the clinical setting. Urology. 2000; 55: 816-9.

4. Trinchieri A, Esposito N, Castelnuovo C: Dissolution of radiolucent renal stones by oral alkalinization with potassium citrate/potassium bicarbonate. Arch Ital Urol Androl. 2009; 81: 188-91.

5. Jackman SV, Potter SR, Regan F, Jarrett TW: Plain abdominal $x$-ray versus computerized tomography screening: sensitivity for stone localization after nonenhanced spiral computerized tomography. J Urol. 2000; 164: 308-10.

6. Johnston R, Lin A, Du J, Mark S: Comparison of kidneyureter-bladder abdominal radiography and computed tomography scout films for identifying renal calculi. BJU Int. 2009; 104: 670-3.

7. Enomoto A, Kimura $\mathrm{H}$, Chairoungdua $A$, Shigeta $Y$, Jutabha $\mathrm{P}$, Cha SH, et al.: Molecular identification of a renal urate anion exchanger that regulates blood urate levels. Nature. 2002; 417: 447-52.

8. Hirasaki S, Koide N, Fujita K, Ogawa H, Tsuji T: Two cases of renal hypouricemia with nephrolithiasis. Intern Med. 1997; 36: 201-5.
9. Dinour D, Gray NK, Ganon L, Knox AJ, Shalev H, Sela BA et al.: Two novel homozygous SLC2A9 mutations cause renal hypouricemia type 2. Nephrol Dial Transplant. 2012; 27: 1035-41.

10. Ekeruo WO, Tan YH, Young MD, Dahm P, Maloney ME, Mathias BJ, et al.: Metabolic risk factors and the impact of medical therapy on the management of nephrolithiasis in obese patients. J Urol. 2004; 172: 159-63.

11. Daudon M, Lacour B, Jungers P: Influence of body size on urinary stone composition in men and women. Urol Res. 2006; 34: 193-9.

12. Maalouf NM, Sakhaee K, Parks JH, Coe FL, Adams-Huet $\mathrm{B}$, Pak CY: Association of urinary $\mathrm{pH}$ with body weight in nephrolithiasis. Kidney Int. 2004; 65: 1422-5.

13. Taylor EN, Curhan GC: Body size and 24-hour urine composition. Am J Kidney Dis. 2006; 48: 905-15.

14. Viprakasit DP, Sawyer MD, Herrell SD, Miller NL: Limitations of ultrasonography in the evaluation of urolithiasis: a correlation with computed tomography. J Endourol. 2012; 26: 209-13.

15. Ray AA, Ghiculete D, Pace KT, Honey RJ: Limitations to ultrasound in the detection and measurement of urinary tract calculi. Urology. 2010; 76: 295-300.

16. Moș C, Holt G, luhasz S, Moș D, Teodor I, Hălbac M: The sensitivity of transabdominal ultrasound in the diagnosis of ureterolithiasis. Med Ultrason. 2010; 12: 188-97.

17. Tartari S, Rizzati R, Righi R, Deledda A, Terrani S, Benea G: Low-dose unenhanced CT protocols according to individual body size for evaluating suspected renal colic: cumulative radiation exposures. Radiol Med. 2010; 115: 105-14.

18. Cicerello E, Merlo F, Maccatrozzo L: Urinary alkalization for the treatment of uric acid nephrolithiasis. Arch Ital Urol Androl. 2010; 82: 145-8.

Correspondence address: Dr. Maneesh Sinha Department of Urology CA6, 15th Main, 11th Cross Padmanabhanagar, Bangalore, 560070, India Fax: + 9180 2639-2693 E-mail: dr.maneesh@nuhospitals.com 